\title{
Latent autoimmune diabetes in adults (LADA)
}

National Diabetes Information Clearinghouse (NDIC)

\section{Definitions}

Diabetes

Defined by National Diabetes Information Clearinghouse (NDIC)

Type 1 diabetes

Defined by National Diabetes Information Clearinghouse (NDIC)

Type 2 diabetes

Defined by National Diabetes Information Clearinghouse (NDIC)

Insulin

Defined by National Diabetes Information Clearinghouse (NDIC)

Injection

Defined by National Diabetes Information Clearinghouse (NDIC)

Antibodies

Defined by National Diabetes Information Clearinghouse (NDIC)

Beta cell

Defined by National Diabetes Information Clearinghouse (NDIC)

\section{Pancreas}

Defined by National Diabetes Information Clearinghouse (NDIC)

Blood glucose level

Defined by National Diabetes Information Clearinghouse (NDIC)

Source

National Diabetes Information Clearinghouse (U.S.). (2009). The diabetes dictionary. [Bethesda, Md.]: U.S. Dept. of Health and Human Services, National Institutes of Health, National Institute of Diabetes and Digestive and Kidney Diseases, National Diabetes Information Clearinghouse.

A type of diabetes, usually first diagnosed after age 30, in which people show signs of both type 1 diabetes and type 2 diabetes. Most people with LADA still produce their own insulin when first diagnosed and do not require insulin injections. Some experts believe that LADA is a slowly developing kind of type 1 diabetes because patients have antibodies against the insulin- producing beta cells in the pancreas. Several years after diagnosis, people with LADA must take insulin to control blood glucose levels. 